\title{
On the Nonexistence of Order Isomorphisms between the Sets of All Self-Adjoint and All Positive Definite Operators
}

\author{
Lajos Molnár \\ MTA-DE "Lendület” Functional Analysis Research Group, Institute of Mathematics, University of Debrecen, P.O. Box 12, \\ Debrecen 4010, Hungary
}

Correspondence should be addressed to Lajos Molnár; molnarl@science.unideb.hu

Received 20 October 2014; Accepted 8 December 2014

Academic Editor: Debora Amadori

Copyright (C) 2015 Lajos Molnár. This is an open access article distributed under the Creative Commons Attribution License, which permits unrestricted use, distribution, and reproduction in any medium, provided the original work is properly cited.

We prove that there is no bijective map between the set of all positive definite operators and the set of all self-adjoint operators on a Hilbert space with dimension greater than 1 which preserves the usual order (the one coming from the concept of positive semidefiniteness) in both directions. We conjecture that a similar assertion is true for general noncommutative $C^{*}$-algebras and present a proof in the finite dimensional case.

\section{Introduction and Results}

It is a trivial fact that the logarithmic function $f(t)=\log t, t>$ 0 , is an order isomorphism from the set $] 0, \infty[$ of positive real numbers onto the real line $]-\infty, \infty[$; that is, it is a bijective map such that

$$
t \leq s \Longleftrightarrow f(t) \leq f(s)
$$

holds for all $t, s \in] 0, \infty[$. In this paper we prove the fact that there is no similar transformation in higher dimensions meaning that there is no such bijective map from the set of all positive definite operators onto the set of all self-adjoint operators on a complex Hilbert space $H$ with $\operatorname{dim} H>1$ which preserves the usual order $\leq$ (the one coming from the concept of positive semidefiniteness) in both directions.

In what follows in a unital $C^{*}$-algebra $\mathscr{A}$ the set of all selfadjoint elements is denoted by $\mathscr{A}_{s}$. An element $A \in \mathscr{A}_{s}$ is called positive semidefinite (here we borrow the terminology of matrix theory rather than that of operator theory where the term "positive" is commonly used) if the spectrum of $A$ lies in $\left[0, \infty\left[\right.\right.$. We use the notation $\mathscr{A}_{+}$for the set of all such elements of $\mathscr{A}_{s}$. The concept of positive semidefiniteness induces an order (more precisely a partial order) $\leq$ on $\mathscr{A}_{s}$. We write $A \leq B$ if $B-A$ is positive semidefinite. The invertible positive semidefinite elements of $\mathscr{A}$ are called positive definite and their set is denoted by $\mathscr{A}_{+}^{-1}$.
For a complex Hilbert space $H, B(H)$ stands for the $C^{*}$ algebra of all bounded linear operators on $H$. The first result of the paper reads as follows.

Theorem 1. Let $H$ be a complex Hilbert space with $\operatorname{dim} H>1$. Then $B(H)_{+}^{-1}$ is not order isomorphic to $B(H)_{s}$; that is, there is no bijective transformation $\phi: B(H)_{+}^{-1} \rightarrow B(H)_{s}$ for which

$$
A \leq B \Longleftrightarrow \phi(A) \leq \phi(B)
$$

holds for any $A, B \in B(H)_{+}^{-1}$.

For the proof of this result we recall the following assertion which describes the structure of all order automorphisms of $B(H)_{+}$. It appeared as Theorem 1 in [1].

Proposition 2. Let $H$ be a complex Hilbert space with $\operatorname{dim} H>1$. Assume $\phi: B(H)_{+} \rightarrow B(H)_{+}$is a bijective map which satisfies

$$
A \leq B \Longleftrightarrow \phi(A) \leq \phi(B)
$$

for any $A, B \in B(H)_{+}$. Then there is an invertible bounded either linear or conjugate linear operator $T$ on $H$ such that $\phi$ is of the form

$$
\phi(A)=T A T^{*}, \quad A \in B(H)_{+} .
$$


Observe that the conclusion in this proposition is obviously not true in the one-dimensional case; there is no similar structural result for all strictly increasing bijections of the interval $[0, \infty[$.

Proof of Theorem 1. For temporary use introduce the following notation. For any $A \in B(H)_{s}$ denote

$$
\begin{gathered}
] 0, A]=\left\{X \in B(H)_{+}^{-1}: X \leq A\right\}, \\
{\left[A, \infty\left[=\left\{X \in B(H)_{s}: X \geq A\right\},\right.\right.} \\
]-\infty, A]=\left\{X \in B(H)_{s}: X \leq A\right\} .
\end{gathered}
$$

Assume now that $\phi$ is an order isomorphism from $B(H)_{+}^{-1}$ onto $B(H)_{s}$, that is, a bijective map $\phi: B(H)_{+}^{-1} \rightarrow B(H)_{s}$ which satisfies (2). Denote $\phi(I)=E, \phi(2 I)=F$. Clearly, $\phi([I, \infty[)=[E, \infty[$, and $\phi(] 0,2 I])=]-\infty, F]$, and the maps $A \mapsto \phi(A+I)-E$ and $A \mapsto-\phi\left((A+(1 / 2) I)^{-1}\right)+F$ are order automorphisms of $B(H)_{+}$. We know the structure of those automorphisms. By Proposition 2 we have invertible bounded either linear or conjugate linear operators $T, S$ on $H$ such that

$$
\begin{aligned}
& \phi(A+I)-E=T A T^{*}, \\
& -\phi\left(\left(A+\left(\frac{1}{2}\right) I\right)^{-1}\right)+F=S A S^{*},
\end{aligned}
$$

$$
A \in B(H)_{+} .
$$

Elementary calculation shows that

$$
\begin{gathered}
\phi(B)=T(B-I) T^{*}+E, \quad B \in[I, \infty[ \\
\left.\left.\phi(B)=-S\left(B^{-1}-\left(\frac{1}{2}\right) I\right) S^{*}+F, \quad B \in\right] 0,2 I\right] .
\end{gathered}
$$

Therefore, it follows that

$$
T B T^{*}=-S B^{-1} S^{*}+G, \quad I \leq B \leq 2 I
$$

holds with some $G \in B(H)_{s}$. In particular, we have $t^{2} T T^{*}=$ $-S S^{*}+t G$ for all real numbers $1 \leq t \leq 2$ which implies $T T^{*}=$ 0 , a clear contradiction.

The proof of Theorem 1 is surprisingly short but let us emphasize that it uses Proposition 2 which is a highly nontrivial result; it tells that the group of order isomorphisms of $B(H)_{+}$is the smallest possible, it consist only of the trivial order automorphisms $A \mapsto T A T^{*}$.

We conjecture that the conclusion in Theorem 1 is valid for every noncommutative $C^{*}$-algebra, too. As for a commutative $C^{*}$-algebra $\mathscr{A}$, we do have an order isomorphism from $\mathscr{A}_{+}^{-1}$ onto $\mathscr{A}_{s}$. In fact, to see this, we recall that $\mathscr{A}$ is isomorphic to an algebra $C(X)$ of all complex valued continuous functions on a compact Hausdorff space $X$, the usual order becoming the pointwise order among functions. Now consider the map $f \mapsto \log \circ f$ which is obviously an order isomorphism from $C(X)_{+}^{-1}$ onto $C(X)_{s}$.

Unfortunately, we do not have a proof for our conjecture concerning general noncommutative $C^{*}$-algebras but in what follows we present a proof in the particular case of finite dimensional $C^{*}$-algebras.

Theorem 3. Let $\mathscr{A}$ be a finite dimensional $C^{*}$-algebra. If there is an order isomorphism between $\mathscr{A}_{+}^{-1}$ and $\mathscr{A}_{s}$, then $\mathscr{A}$ is necessarily commutative.

The proof of this theorem is very similar in spirit to that of Theorem 1. However, to carry it out we need some auxiliary results. To begin with, we first recall that it is well known that any finite dimensional $C^{*}$-algebra $\mathscr{A}$ is isomorphic to a finite direct sum of full operator algebras over finite dimensional Hilbert spaces. So we may and do assume that we have positive integers $n_{1}, \ldots, n_{m}$ such that

$$
\mathscr{A}=B\left(\mathbb{C}^{n_{1}}\right) \oplus \cdots \oplus B\left(\mathbb{C}^{n_{m}}\right)
$$

Now, the main auxiliary result follows which describes the structure of all order automorphisms of $\mathscr{A}_{s}$.

Theorem 4. Let $\phi$ be an order automorphism of the set $\mathscr{A}_{+}=$ $B\left(\mathbb{C}^{n_{1}}\right)_{+} \oplus \cdots \oplus B\left(\mathbb{C}^{n_{m}}\right)_{+}$of all positive semidefinite elements of the $C^{*}$-algebra $\mathscr{A}$ in (9). Then there is a permutation $\varphi$ of the set $\{1, \ldots, m\}$ such that $n_{i}=n_{\varphi(i)}, i=1, \ldots, m$ and we have

$$
\begin{array}{r}
\phi\left(A_{1}, \ldots, A_{m}\right)=\left(\phi_{\varphi(1)}\left(A_{\varphi(1)}\right), \ldots, \phi_{\varphi(m)}\left(A_{\varphi(m)}\right)\right), \\
A_{i} \in B\left(\mathbb{C}^{n_{i}}\right)_{+}, \quad i=1, \ldots, m,
\end{array}
$$

where for any $i \in\{1, \ldots, m\}$ with $n_{i}>1$ there is an invertible either linear or conjugate linear operator $T_{i}$ on $\mathbb{C}^{n_{i}}$ such that

$$
\phi_{i}\left(A_{i}\right)=T_{i} A_{i} T_{i}^{*}, \quad A_{i} \in B\left(\mathbb{C}^{n_{i}}\right)_{+}
$$

while for any $i \in\{1, \ldots, m\}$ with $n_{i}=1$ we have a strictly increasing bijection $f_{i}$ of the interval $[0, \infty[$ such that

$$
\phi_{i}\left(A_{i}\right)=f_{i}\left(A_{i}\right), \quad A_{i} \in B\left(\mathbb{C}^{n_{i}}\right)_{+}=[0, \infty[.
$$

In the proof of this theorem we will need the following statement about the structure of all Jordan $*$-automorphisms of finite dimensional $C^{*}$-algebras. The result is no doubt very well known but we still could not find any authentic reference in the literature. Hence, to make our paper self-contained, we present it with a short complete proof. Recall that a Jordan *-homomorphism from a $*$-algebra $\mathscr{R}$ into another one $\mathscr{R}^{\prime}$ is a linear map $\phi: \mathscr{R} \rightarrow \mathscr{R}^{\prime}$ which satisfies $\phi\left(A^{2}\right)=\phi(A)^{2}$ for all $A \in \mathscr{R}$, or equivalently, $\phi(A B+B A)=\phi(A) \phi(B)+$ $\phi(B) \phi(A)$ holds for all $A, B \in \mathscr{R}$, and $\phi$ also fulfills $\phi\left(A^{*}\right)=$ $\phi(A)^{*}, A \in \mathscr{R}$. A Jordan $*$-isomorphism is a bijective Jordan *-homomorphism.

Proposition 5. Let $\phi$ be a Jordan *-automorphism of the $C^{*}$ algebra $\mathscr{A}=B\left(\mathbb{C}^{n_{1}}\right) \oplus \cdots \oplus B\left(\mathbb{C}^{n_{m}}\right)$. Then there is a permutation $\varphi$ of $\{1, \ldots, m\}$ such that $n_{i}=n_{\varphi(i)}, i=1, \ldots, m$ and we have

$$
\begin{array}{r}
\phi\left(A_{1}, \ldots, A_{m}\right)=\left(\phi_{\varphi(1)}\left(A_{\varphi(1)}\right), \ldots, \phi_{\varphi(m)}\left(A_{\varphi(m)}\right)\right), \\
A_{i} \in B\left(\mathbb{C}^{n_{i}}\right), \quad i=1, \ldots, m,
\end{array}
$$


where for any $i=1, \ldots, m$ the map $\phi_{i}$ is either of the form

$$
\phi_{i}\left(A_{i}\right)=U_{i} A_{i} U_{i}^{*}, \quad A_{i} \in B\left(\mathbb{C}^{n_{i}}\right)
$$

with a unitary $U_{i}$ on $\mathbb{C}^{n_{i}}$ or of the form

$$
\phi_{i}\left(A_{i}\right)=U_{i} A_{i}^{*} U_{i}^{*}, \quad A_{i} \in B\left(\mathbb{C}^{n_{i}}\right)
$$

with an antiunitary $U_{i}$ on $\mathbb{C}^{n_{i}}$.

Proof. The elements of $\mathscr{A}=B\left(\mathbb{C}^{n_{1}}\right) \oplus \cdots \oplus B\left(\mathbb{C}^{n_{m}}\right)$ are $m$-tuples having $i$ th component in the $i$ th coordinate space $B\left(\mathbb{C}^{n_{i}}\right)$. The set of all $m$-tuples whose components are all zero except for the $i$ th one is called the $i$ th factor in the decomposition of $\mathscr{A}$.

Clearly, $\phi$ maps projections to projections. We learn from $[2,6.3 .2$. Lemma] that just as any Jordan homomorphism, $\phi$ necessarily preserves the Jordan triple product, that is, satisfies $\phi(A B A)=\phi(A) \phi(B) \phi(A), A, B \in \mathscr{A}$. It follows from elementary computations (see Lemma 2 in [3]) that in any $C^{*}$-algebra the projections $P, Q$ commute if and only if $P Q P$ is a projection. We deduce that $\phi$ preserves commuting projections in both directions. Since $\mathscr{A}$ is linearly generated by its projections, we obtain that $\phi$ sends the set of all central projections onto itself. It is apparent that $\phi$ preserves the order among projections in both directions. We then infer that $\phi$ sends the set of all nonzero minimal central projections onto itself. A factor in the decomposition of $\mathscr{A}$ can be characterized as the set of all elements $A \in$ $\mathscr{A}$ for which $A P=P A=A$ holds with a given nonzero minimal central projection $P$ in $\mathscr{A}$. This equality is clearly equivalent to that $\phi(A) \phi(P)=\phi(P) \phi(A)=\phi(A)$ which holds for all $A \in \mathscr{A}$ meaning that $\phi$ maps a factor in the decomposition of $\mathscr{A}$ onto another (not necessarily different) factor. Since the different nonzero minimal projections are clearly orthogonal (i.e., they have zero product), the same holds for their images and this implies that different factors are mapped onto different factors. Therefore, $\phi$ is the direct sum of Jordan $*$-isomorphisms $\psi_{i}$ from $B\left(\mathbb{C}^{n_{i}}\right)$ onto $B\left(\mathbb{C}^{n_{\varphi(i)}}\right)$, $i=1, \ldots, m$, where $\varphi:\{1, \ldots, m\} \rightarrow\{1, \ldots, m\}$ is a map. We trivially obtain that $\varphi$ is a permutation of $\{1, \ldots, m\}$ and that $n_{i}=n_{\varphi(i)}$ holds for all $i$. Therefore, $\psi_{i}$ is a Jordan *-automorphism of $B\left(\mathbb{C}^{n_{i}}\right)$. Since these algebras are prime, by $\left[2,6.3 .7\right.$ Theorem] we obtain that every $\psi_{i}$ is either a $*$ automorphism or a $*$-antiautomorphism. The structure of $*$ automorphisms of full operator algebras are well known to be implemented by unitary operators (meaning that they are of the form $A \mapsto U A U^{*}$ with a unitary $U$ ) from which one can easily deduce that the $*$-antiautomorphisms are of the form $A \mapsto U A^{*} U^{*}$ with an antiunitary operator $U$. The proof can be completed trivially.

We are now in a position to prove Theorem 4.

Proof of Theorem 4. Let us introduce some notion for temporary use. First recall that in the $m$-tuple $\underline{A}=\left(A_{1}, \ldots, A_{m}\right) \in$ $\mathscr{A}$, the element $A_{i}$ is said to be the component in the $i$ th coordinate space. If $n_{i}=1$ then we say that it is a commutative coordinate space and $A_{i}$ is called a commutative component. We define the rank of an element $\underline{A}=\left(A_{1}, \ldots, A_{m}\right) \in \mathscr{A}$ by the sum of the ranks of its components as linear operators.
Clearly, the order in $\mathscr{A}_{+}=B\left(\mathbb{C}^{n_{1}}\right)_{+} \oplus \cdots \oplus B\left(\mathbb{C}^{n_{m}}\right)_{+}$is meant componentwise.

Now, we essentially follow the proof of Theorem 1 in [1]. Indeed, first prove that $\phi$ preserves the rank. To see this, observe that we clearly have $\phi(\underline{0})=\underline{0}$. The rank-one elements in $\mathscr{A}_{+}$can easily be characterized as the nonzero elements $\underline{A}$ for which we have that for any $\underline{0} \leq \underline{B}, \underline{C} \leq \underline{A}$ either $\underline{B} \leq \underline{C}$ or $\underline{C} \leq \underline{B}$ holds. Therefore, $\underline{A} \in \mathscr{A}_{+}$is of rank one if and only if $\phi(\underline{A})$ is of rank one. Assume the same holds for the ranks $1, \ldots, k$. We are going to show that then $\phi$ has the corresponding property concerning the rank $(k+1)$, too. One can easily check the following characterization of elements of rank greater than $k+1$ (cf. Lemma 2 in [1]). The element $\underline{A} \in \mathscr{A}_{+}$is of rank greater than $k+1$ if and only if there are elements $\underline{B}, \underline{C} \in \mathscr{A}_{+}$with rank $k$ and rank greater than 1, respectively, such that $\underline{B}, \underline{C} \leq \underline{A}$ and there is no nonzero element $\underline{D} \in \mathscr{A}_{+}$such that $\underline{D} \leq \underline{B}$, $\underline{C}$. Using this characterization we deduce that $\phi$ sends the set of rank- $(k+1)$ elements onto itself. Consequently, $\phi$ preserves the rank.

We next show that $\phi$ maps factors corresponding to commutative coordinate spaces onto factors of the same kind. To see this, we first assert the following: for any $A \in$ $B\left(\mathbb{C}^{l}\right)_{+}$the infimum of $A$ exists in $B\left(\mathbb{C}^{l}\right)_{+}$, with every $X \in$ $B\left(\mathbb{C}^{l}\right)_{+}$(meaning that the set $\{A, X\}$ has infimum in $B\left(\mathbb{C}^{l}\right)_{+}$ for every $X \in B\left(\mathbb{C}^{l}\right)_{+}$) if and only if the rank of $A$ is at most one. Indeed, we deduce this characterization from the result Corollary 3.12 in the paper [4] of Moreland and Gudder. To be precise, their result says that in the set of all positive semidefinite operators on a finite dimensional space with eigenvalues less than or equal to 1 , an element $A$ has infimum with any other element if and only if $A$ is either a projection or a matrix of rank at most 1 . But in our case there is a serious difference, namely, the eigenvalues are not bounded from above. So, we argue as follows. If $A$ is a rank-one positive semidefinite operator and $X$ is an arbitrary positive semidefinite operator, then multiplying them by a suitable positive constant (observe that this multiplication as a transformation is an order isomorphism) we can assume that the eigenvalues of $A, X$ are bounded by 1 and hence obtain that their infimum does exist. On the other hand, if $A$ is a positive semidefinite operator which has rank at least two, its eigenvalues are bounded by a positive constant $c$, and it has infimum with every other positive semidefinite operator $X$ with eigenvalues not greater than $c$, then applying the result of Moreland and Gudder we infer that $A$ is a projection multiplied by $c$. But $c$ can clearly vary and hence we immediately arrive at a contradiction. This proves the desired characterization of rank-one elements in $B\left(\mathbb{C}^{l}\right)_{+}$.

After this, one can easily verify that the nonzero component of a nonzero rank-one element $\underline{A}$ of $\mathscr{A}_{+}$belongs to a commutative coordinate space if and only if for every ranktwo element $\underline{B} \in \mathscr{A}_{+}$with $\underline{B} \geq \underline{A}$ we have that $\underline{B}$ has infimum in $\mathscr{A}_{+}$with all elements $\underline{X} \in \mathscr{A}_{+}$. Due to this characterization the rank-one elements with nonzero components in a commutative coordinate space are mapped into elements of the same type. Clearly all such elements with nonzero component in the same commutative coordinate space are all comparable. This gives us that a factor corresponding to 
a commutative coordinate space is mapped into a factor of the same type. Since $\phi^{-1}$ has the same preserver properties as $\phi$, it follows that $\phi$ in fact maps such factors onto such factors. Again, using the comparability reasoning, we see that $\phi$ permutes the commutative coordinate spaces among themselves and clearly acts on each of them as a strictly increasing bijection of the real interval $[0, \infty[$. Let us now consider the noncommutative coordinate spaces. We claim that the restriction of our transformation onto the set of all elements of $\mathscr{A}_{+}$with zero commutative components maps that set onto itself. In fact, if the image $\phi(\underline{A})$ of such an element $\underline{A}$ has a nonzero commutative component, then we can find a rank-one element $R$ with nonzero commutative component which is below $\phi(\underline{A})$; that is, $\underline{R} \leq \phi(\underline{A})$ holds. Taking preimage we would obtain that the rank-one element $\phi^{-1}(\underline{R})$ with a nonzero commutative component is below our original element $\underline{A}$ which has commutative components all zero. This is a clear contradiction. Therefore, the so restricted transformation can be considered as an order automorphism on the direct sum of full sets of all positive semidefinite operators on Hilbert spaces with dimension at least 2.

From this point on we can literally follow our argument given on the pages 5907-5908 in [1] using a beautiful result of Rothaus [5] and verify that the above transformation is additive and positive homogeneous and hence it can be extended to a linear order isomorphism on the direct sum of the corresponding full operator algebras. Still following the argument given in [1] we apply a theorem of Kadison [6] to see that this map is a Jordan $*$-automorphism of the underlying algebra followed by multiplication with a fixed positive definite element from both sides. The structure of Jordan *-automorphisms of the algebras under consideration is described in Proposition 5. Therefore, we know the structure of the order isomorphism $\phi$ on the direct sum of the noncommutative factors as well as on the direct sum of the commutative ones. In fact, we see that $\phi$ is of the required form on the set of elements having only commutative nonzero components as well as on the set of elements having only noncommutative nonzero components. But an arbitrary element of $\mathscr{A}_{+}$is the supremum of two such elements (one of the former type and one of the latter type) and since $\phi$ preserves supremum (whenever it exists), we complete the proof of the theorem.

After this preparation we can now easily prove our second main result Theorem 3 .

Proof of Theorem 3. Assume, on the contrary, that in the decomposition $\mathscr{A}=B\left(\mathbb{C}^{n_{1}}\right) \oplus \cdots \oplus B\left(\mathbb{C}^{n_{m}}\right)$ we have, for example, $n_{1} \neq 1$ and there exists an order isomorphism $\phi$ from $\mathscr{A}_{+}^{-1}$ onto $\mathscr{A}_{s}$. We apply an argument similar to the proof of Theorem 1 to reach a contradiction. Namely, define the order automorphisms $\underline{A} \mapsto \phi(\underline{A}+\underline{I})-\phi(\underline{I})$ and $\underline{A} \mapsto-\phi\left((\underline{A}+(1 / 2) \underline{I})^{-1}\right)+\phi(2 \underline{I})$ of $\mathscr{A}_{+}$. Apply the structural result Theorem 4 for these maps and consider only the first components of their images. Just as in the proof of Theorem 1 we deduce the identity

$$
T_{i} B_{i} T_{i}^{*}=-S_{j} B_{j}^{-1} S_{j}^{*}+G_{i j}, \quad \underline{I} \leq \underline{B} \leq 2 \underline{I}
$$

for some given indices $i, j \in\{1, \ldots, m\}$ where $T_{i}, S_{j}$ are invertible either linear or conjugate linear operators and $G_{i j}$ is a self-adjoint operator. We clearly have $i=j$ and then, exactly like in that proof, we arrive at a contradiction. This verifies the statement.

Remark 6. We conclude the paper with a few remarks. First of all we note that one could consider a seemingly more general problem. To formulate it, take a $C^{*}$-algebra $\mathscr{A}$ and for any interval $I \subset \mathbb{R}$ define $\mathscr{A}(I)$ as the set of all elements in $\mathscr{A}_{s}$ with spectrum contained in $I$. The existence of an order isomorphism (with respect to the usual order) among pairs of sets $\mathscr{A}(I), \mathscr{A}(J)$ defines an equivalence relation on the collection of all those sets. Now one may pose the problem that, considering all open intervals of the real line, how many equivalence classes we have. However, the fact is that one does not obtain anything essentially new by raising that question. The reason is the following. Clearly, for any two bounded open intervals $I, J \subset \mathbb{R}$ the corresponding sets $\mathscr{A}(I), \mathscr{A}(J)$ are order isomorphic (via a simple affine transformation on $\mathscr{A})$. Next, the map $A \mapsto-(A-I)^{-1}-I$ gives an order isomorphism between $\mathscr{A}(] 0,1[)$ and $\mathscr{A}(] 0, \infty[)$ and this latter set is obviously order isomorphic to any $\mathscr{A}(] a, \infty[), a \in \mathbb{R}$. Furthermore, the map $A \mapsto-A^{-1}$ is an order isomorphism from $\mathscr{A}(] 0, \infty[)$ onto $\mathscr{A}(]-\infty, 0[)$ and this latter set is order isomorphic to any $\mathscr{A}(]-\infty, b[), b \in \mathbb{R}$. Consequently, the sets $\mathscr{A}(I)$ for any proper open interval $I \subset \mathbb{R}$ are in the same equivalence class. Hence the only interesting question is to explore if for any proper open interval $I$, say, for $I=] 0, \infty[$ we have that $\mathscr{A}(I)$ is order isomorphic to $\mathscr{A}(J), J=]-\infty, \infty[$. But this is exactly the problem we have treated above in the case where $\mathscr{A}=B(H)$ and formulated a conjecture for general $C^{*}$-algebras $\mathscr{A}$.

Apparently, one can go further and consider all sorts of intervals, not only the open ones. Clearly, there is no order isomorphism between $\mathscr{A}(I)$ and $\mathscr{A}(J)$ if one of the intervals $I, J$ is open but the other one is not. That means that we then have new equivalence classes. Actually, we have three of them which are easily seen to correspond to all bounded closed intervals, to all intervals of one of the two forms $[a, b[,[a, \infty[$, $a, b \in \mathbb{R}$, and to all intervals of one of the two forms $] a, b]$, ]$\infty, b], a, b \in \mathbb{R}$, respectively.

Above we have discussed the question of the existence of order isomorphisms among pairs of sets $\mathscr{A}(I), \mathscr{A}(J)$. One might want to get further and be interested in the structure of all order isomorphisms between the sets $\mathscr{A}(I), \mathscr{A}(J)$ which belong to the same equivalence class. Since within one equivalence class we can easily find order isomorphisms between different elements (see above), the question reduces to the problem of describing all order automorphisms of one particular element in that class. Let us consider the case where $\mathscr{A}=B(H), \operatorname{dim} H>1$. We already know that there are five equivalence classes. As for the interval $I=]-\infty, \infty[$, the structure of order automorphisms of the corresponding set has been described in Theorem 2 in [1]. Concerning the interval $I=] 0, \infty[$, similar result was obtained in Theorem 1 in [7]. As for $I=[0, \infty$ [, we refer to Theorem 1 in [1] which we have reformulated in Proposition 2. Concerning the interval 
$I=]-\infty, 0]$, the description of all order automorphisms can easily be deduced from the one relating to the previous interval $[0, \infty[$. So, only one question remains, the one that concerns the closed unit interval $[0,1]$. In fact, this problem proved to be very deep and it has recently been solved by Šemrl. For his corresponding result see Corollary 1.2 in [8] (we also refer to Theorem 3.1 in [9]). All the results that have just been mentioned concern the particular $C^{*}$-algebra $B(H)$. We close this paragraph by telling that as for the case of general $C^{*}$-algebras $\mathscr{A}$, we do not see hope to obtain similar precise descriptions of the order automorphism groups of the related sets.

Now we turn back to the question of the nonexistence of order isomorphisms between $\mathscr{A}(] 0, \infty[)$ and $\mathscr{A}(]-\infty, \infty[)$. Above we have formulated the conjecture that such a transformation exists only if $\mathscr{A}$ is commutative. In fact, we believe that even more is true: we suspect that if we have a bijective map $\phi: \mathscr{A}(]-\infty, \infty[) \rightarrow \mathscr{A}(] 0, \infty[)$ with the property that

$$
A \leq B \Longrightarrow \phi(A) \leq \phi(B)
$$

holds for any $A, B \in \mathscr{A}(]-\infty, \infty[)$, then $\mathscr{A}$ is necessarily commutative. A few comments on this modified conjecture follow. First, we refer to the paper [10] where it was shown that if the exponential function on the interval $[0, \infty$ [ is monotone increasing relative to a given $C^{*}$-algebra $\mathscr{A}$ (meaning that for any $A, B \in \mathscr{A}_{s}$ with $0 \leq A \leq B$ we have $\exp (A) \leq$ $\exp (B)$ ), then the algebra $\mathscr{A}$ is commutative. In [11] this result was extended for any continuous monotone increasing and nonconcave real function on $[0, \infty[$ in the place of the exponential function. However, it is apparent that a nonconstant continuous monotone increasing function from ] $-\infty, \infty[$ to ] $0, \infty[$ cannot be concave. Therefore, it follows easily that if there is a nonconstant continuous monotone increasing function $f:]-\infty, \infty[\rightarrow] 0, \infty[$ with the property that

$$
A \leq B \Longrightarrow f(A) \leq f(B)
$$

holds for any $A, B \in \mathscr{A}(]-\infty, \infty[)$, then $\mathscr{A}$ is necessarily commutative. It is important to point out here that $f(A)$ originates from the continuous function calculus and $f$ is a real function and not a transformation on $\mathscr{A}_{s}$. Therefore, the above argument does not prove our conjecture at all; it only gives some hope that it might be true.

To finish our remarks, we recall that above we have dealt with the usual order $\leq$ among the self-adjoint elements of a $C^{*}$-algebra. Quite naturally, one may ask what happens if we consider other orders. Probably the most well-known order on the operator space $B(H)_{s}$ which is different from $\leq$ is the one called spectral order. It was defined by Olson [12] in terms of the spectral resolution of operators as follows. Let $A, B \in$ $B(H)_{s}$ with corresponding spectral measures $E_{A}, E_{B}$ defined on the Borel subsets of $\mathbb{R}$. We write

$$
\left.\left.\left.\left.A \preceq B \quad \text { iff } E_{A}(]-\infty, t\right]\right) \geq E_{B}(]-\infty, t\right]\right), t \in \mathbb{R} .
$$

The relation $\preceq$ called spectral order is a partial order on $B(H)_{s}$. The main result in [12] says that every nonempty subset of $B(H)_{s}$ which has an upper bound necessarily has supremum (a similar assertion is valid concerning the existence of the infimum). This is the property which makes the spectral order so useful. In fact, we recall the surprising result of Kadison presented in [13] that $B(H)_{s}$ equipped with the usual order $\leq$ is a so-called antilattice: for any $A, B \in B(H)_{s}$, the supremum of the set $\{A, B\}$ exists if and only if $A, B$ are comparable, that is, if we have either $A \leq B$ or $B \leq A$. This strange property of the order $\leq$ motivated Olson to introduce the spectral order on $B(H)_{s}$. Now, if we consider our starting isomorphism problem (see Theorem 1) for the spectral order, the question is just easy. For any continuous bijective monotone increasing function $f:]-\infty, \infty[\rightarrow] 0, \infty[$, the transformation $A \mapsto$ $f(A)$ is apparently a spectral order isomorphism from $B(H)_{s}$ onto $B(H)_{+}^{-1}$.

\section{Conflict of Interests}

The author declares that there is no conflict of interests regarding the publication of this paper.

\section{Acknowledgment}

The author was supported by the "Lendület" Program (LP2012-46/2012) of the Hungarian Academy of Sciences.

\section{References}

[1] L. Molnár, "Order-automorphisms of the set of bounded observables," Journal of Mathematical Physics, vol. 42, no. 12, pp. 5904-5909, 2001.

[2] T. W. Palmer, Banach Algebras and the General Theory of *Algebras, vol. 1 of Encyclopedia of Mathematics and Its Applications 49, Cambridge University Press, 1994.

[3] R. Beneduci and L. Molnár, "On the standard K-loop structure of positive invertible elements in a $C^{*}$-algebra," Journal of Mathematical Analysis and Applications, vol. 420, no. 1, pp. 551562, 2014.

[4] T. Moreland and S. Gudder, "Infima of Hilbert space effects," Linear Algebra and Its Applications, vol. 286, no. 1-3, pp. 1-17, 1999.

[5] O. S. Rothaus, "Order isomorphisms of cones," Proceedings of the American Mathematical Society, vol. 17, pp. 1284-1288, 1966.

[6] R. V. Kadison, "A generalized Schwarz inequality and algebraic invariants for operator algebras," Annals of Mathematics. Second Series, vol. 56, pp. 494-503, 1952.

[7] L. Molnár, "Order automorphisms on positive definite operators and a few applications," Linear Algebra and Its Applications, vol. 434, no. 10, pp. 2158-2169, 2011.

[8] P. Šemrl, "Comparability preserving maps on Hilbert space effect algebras," Communications in Mathematical Physics, vol. 313, no. 2, pp. 375-384, 2012.

[9] P. Šemrl, "Symmetries of Hilbert space effect algebras," Journal of the London Mathematical Society. Second Series, vol. 88, no. 2, pp. 417-436, 2013.

[10] W. Wu, "An order characterization of commutativity for $C^{*}$ algebras," Proceedings of the American Mathematical Society, vol. 129, no. 4, pp. 983-987, 2001.

[11] M. Nagisa, M. Ueda, and S. Wada, "Commutativity of operators," Nihonkai Mathematical Journal, vol. 17, no. 1, pp. 1-8, 2006. 
[12] M. P. Olson, "The selfadjoint operators of a von Neumann algebra form a conditionally complete lattice," Proceedings of the American Mathematical Society, vol. 28, pp. 537-544, 1971.

[13] R. V. Kadison, "Order properties of bounded self-adjoint operators," Proceedings of the American Mathematical Society, vol. 2, pp. 505-510, 1951. 


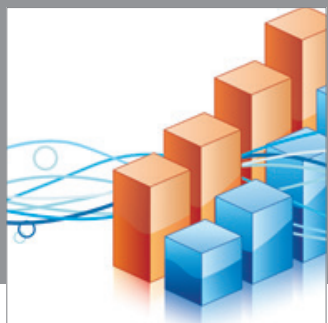

Advances in

Operations Research

mansans

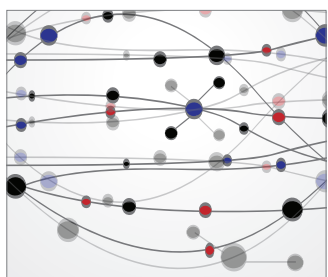

The Scientific World Journal
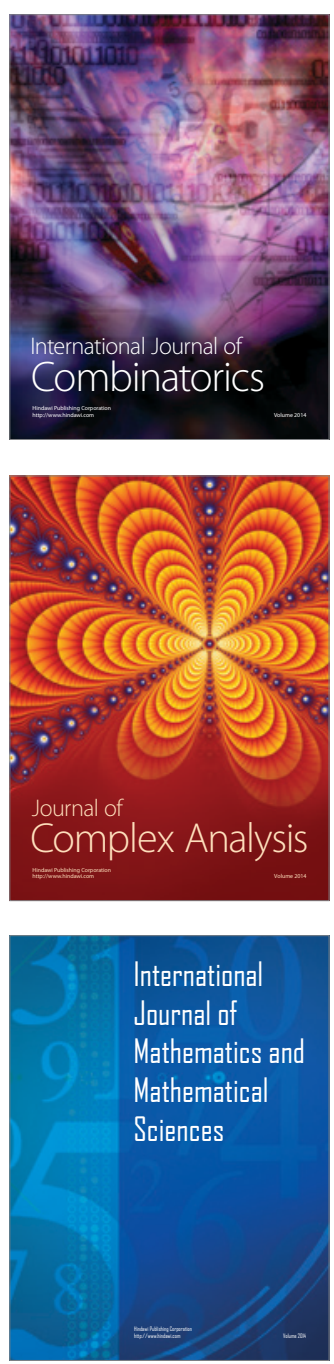
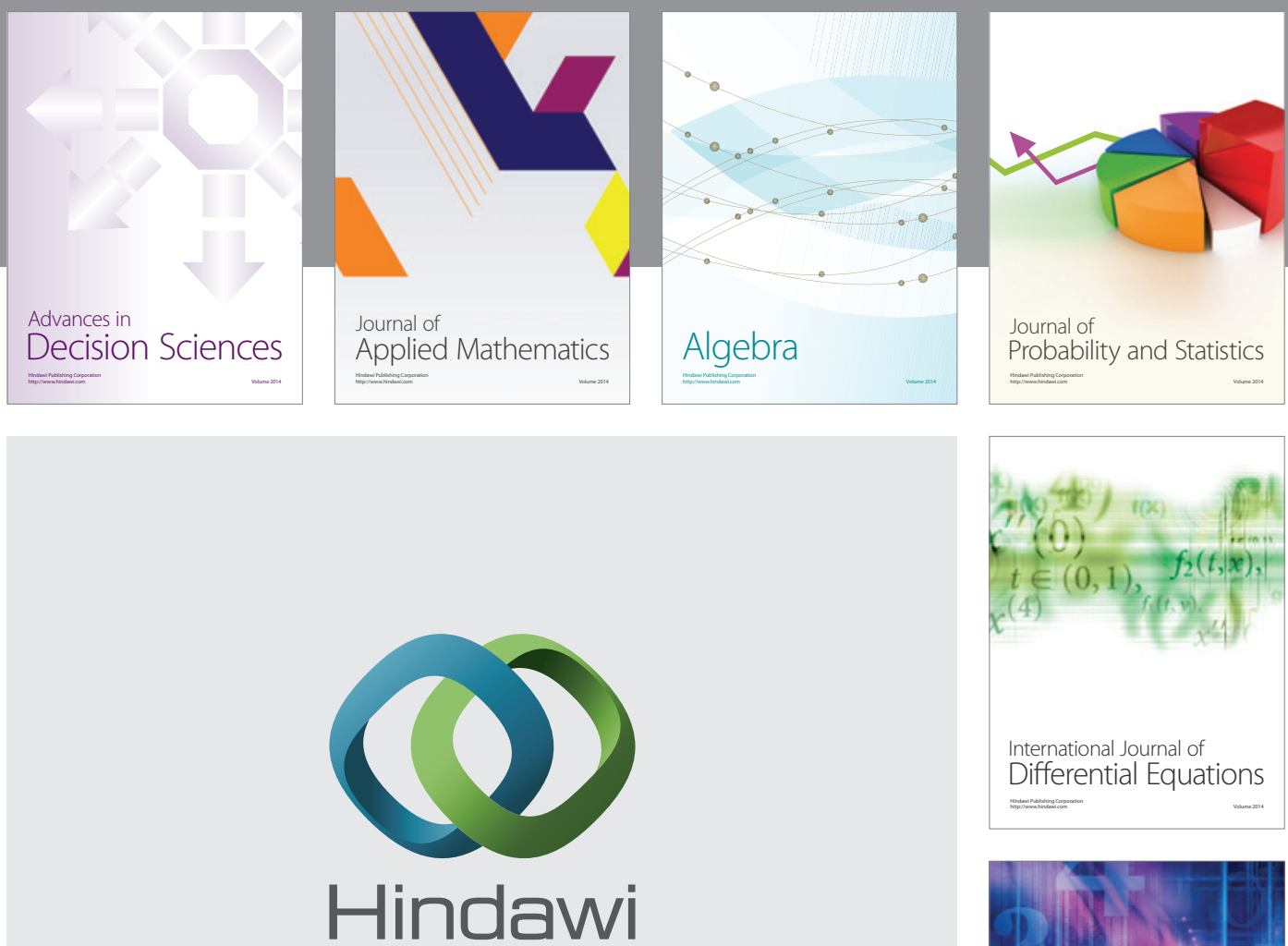

Submit your manuscripts at http://www.hindawi.com
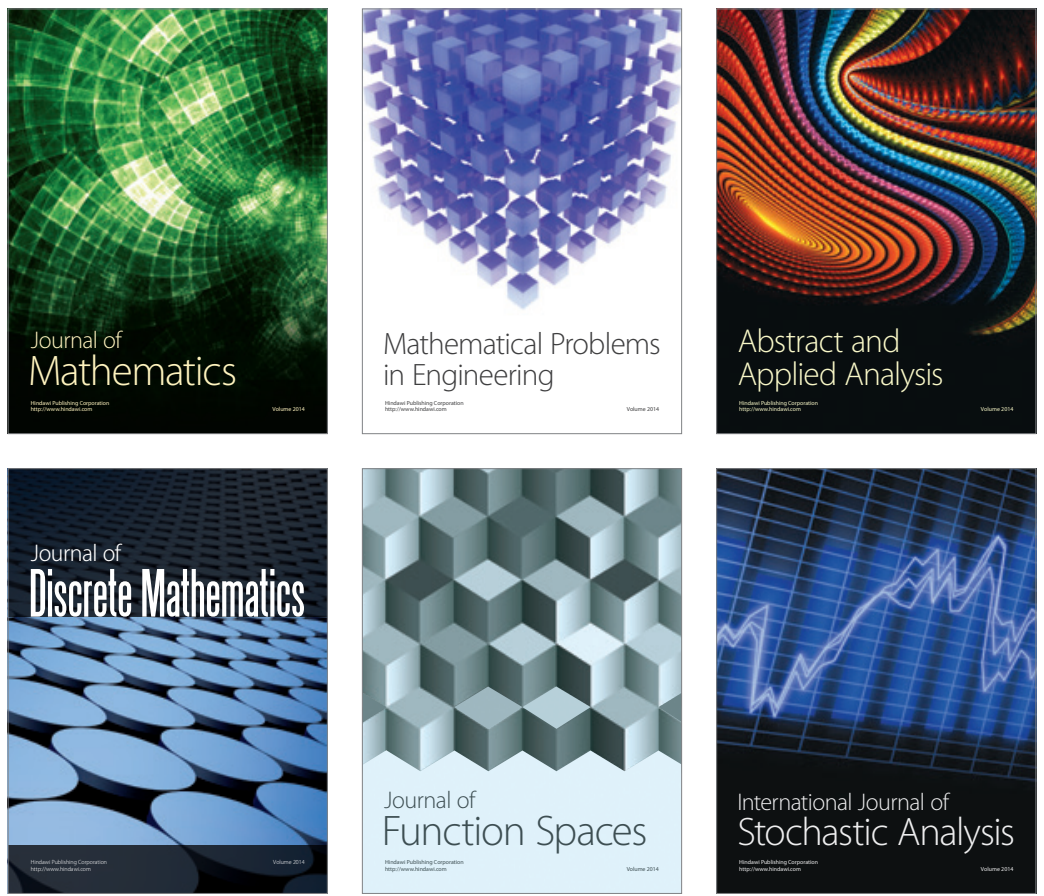

Journal of

Function Spaces

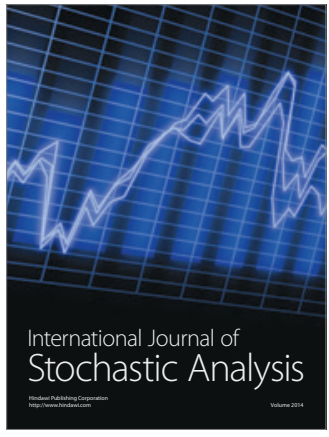

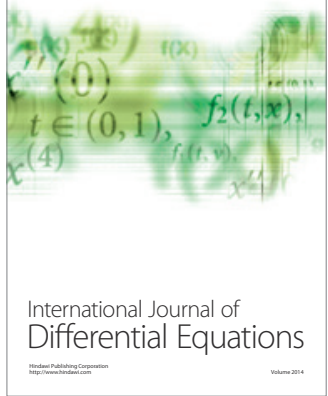
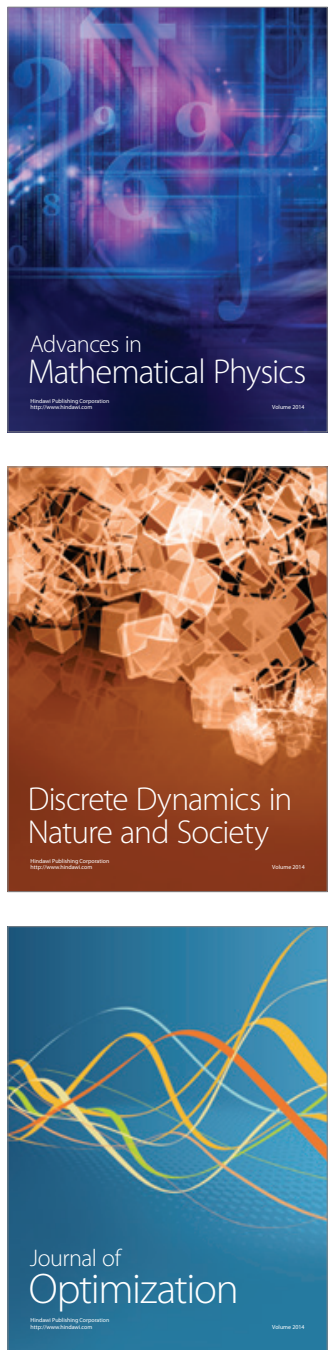